\title{
Bone marrow fibrosis as prognostic marker in adult patients with acute lymphoblastic leukemia
}

\author{
Ana Cooke ${ }^{1}$ - Daniel Montante-Montes ${ }^{1} \cdot$ Diego Zúñiga-Tamayo $^{1} \cdot$ Mauricio Rivera $^{2} \cdot$ Christianne Bourlon $^{1}$. \\ Álvaro Aguayo ${ }^{1} \cdot$ Roberta Demichelis-Gómez $^{1}$
}

Received: 6 January 2019 / Accepted: 12 April 2019 / Published online: 9 May 2019

(C) Springer-Verlag GmbH Germany, part of Springer Nature 2019

\begin{abstract}
The role of bone marrow (BM) fibrosis on outcomes of children with acute lymphoblastic leukemia (ALL) has been described with inconsistent results. In adults, there is no information regarding its significance. The aim of this study was to describe the prognostic impact of BM fibrosis in this group of patients. We included 88 biopsies of 44 adult patients. Reticulin and Masson trichrome stains were performed. Fibrosis was graded by modified Bauermeister scale and volume of reticulin/collagen fibers, both methods had good correlation. Survival analyses were performed regarding (1) the presence or not of significant fibrosis at diagnosis, (2) the reduction or not of fibrosis post-treatment, and (3) the amount of fibrosis at diagnosis combined with the depth of the reduction post-treatment. Sixty-six percent of the patients had significant fibrosis at diagnosis (Bauermeister $\geq$ grade 2 ) and $45 \%$ achieved a significant reduction $(\geq 50 \%$ volume of reticulin/collagen fibers) post-treatment. The presence of fibrosis at diagnosis had no clear impact on outcomes; however, a significant reduction of the fibrosis post-treatment in patients with standard-risk ALL was correlated with longer RFS (19.1 months vs 10.6 months, $p=.022)$ and OS (54.4 months vs 16.4 months, $p=.033)$. Patients who had fibrosis at diagnosis combined with a deep reduction post-treatment had a better RFS and OS compared with those who did not have fibrosis at diagnosis regardless of its reduction or those who did not achieve a deep reduction. To our knowledge, this is the first study to describe an impact of BM fibrosis reduction post-treatment in adult patients with ALL.
\end{abstract}

Keywords Acute lymphoblastic leukemia $\cdot$ Fibrosis $\cdot$ Reticulin $\cdot$ Collagen $\cdot$ Trichrome stain $\cdot$ Biopsy

\section{Introduction}

Acute lymphoblastic leukemia (ALL) is a hematologic disease characterized by uncontrolled proliferation of immature lymphoid cells. Its incidence varies between countries. In the

Submission declaration and verification This work was previously presented as an abstract at the European School of Hematology Clinical Updates on Acute Leukemias (Budapest 2018) meeting and at the Society of Hematologic Oncology 6th annual meeting.

Roberta Demichelis-Gómez

robertademichelis@gmail.com

1 Hematology and Oncology Department, Instituto Nacional de Ciencias Medicas y Nutricion Salvador Zubiran, Vasco de Quiroga 15, Sección XVI Belisario Domínguez, Tlalpan, 14080 Mexico City, Mexico

2 Faculty of Medicine, Universidad Autonoma de Coahuila, Ciudad Universitaria Arteaga, Blvd. V. Carranza s/n Col. República Oriente, 25280 Saltillo, Coahuila, Mexico
USA, an incidence of 1.7 cases per 100,000 person-years [1] has been reported; in Latin America, the incidence is higher with up to 2.1 cases per 100,000 person-years [2]. In the last decade, survival rates in children have improved, with a 5year overall survival (OS) approaching $90 \%[2,3]$; however, this has not been reproduced in adults. According to age group, 5-year OS for adolescents and young adults (AYA) is $42-63 \%, 24 \%$ in patients between 40 and 59 years, and $17 \%$ in those older than 60 years $[4,5]$.

It is known that bone marrow (BM) fibrosis is commonly found in patients with acute leukemia [6] and that this fibrosis reduces significantly after treatment [7-11]. Previous studies have described the role of BM microenvironment and fibrosis as a prognostic marker in ALL with contradictory results. Some clinical studies propose that fibrosis serves as a sanctuary for blasts, reducing response to chemotherapy [12, 13], and others have associated BM fibrosis with lower white blood cell and peripheral blast counts, characteristics classically associated with better prognosis $[14,15]$. 
Norén-Nyström et al. in Sweden is the only group that has consistently reported the prognostic role of BM fibrosis and vascular density in pediatric patients with ALL. In high-risk patients, they described more BM fibrosis and vascular density at diagnosis, and this was related to higher rates of postinduction positive minimal residual disease and shorter progression-free survival (PFS). In addition, a rapid and greater than $20 \%$ reduction in post-induction BM fibrosis was associated with longer relapse-free survival (RFS). They did not find any association between BM fibrosis and specific demographic characteristics, immunophenotype, karyotype, or a prognostic role in standard-risk patients. [16-18]

To our knowledge, there are no studies describing the prognostic role of BM fibrosis on the survival outcomes of adult patients with ALL. The aim of this study is to describe the impact of BM fibrosis at diagnosis and its reduction postinduction treatment on induction failure, RFS, and OS in adult patients with ALL.

\section{Materials and methods}

\section{Patients}

From February 2011 to February 2016, we retrospectively collected demographic information, clinical data, and outcomes from clinical records of patients. We included patients aged $\geq 18$ years, consecutively diagnosed with ALL, treated, and followed at our institution, with available and goodquality BM biopsies on diagnosis and post-induction treatment, and who had no previous cancer or disease known to cause BM fibrosis. This protocol was approved by our local ethical committee.

ALL diagnosis was established according to the 2016 World Health Organization (WHO) criteria [19, 20]. Risk was stratified according to classic risk factors ( $>35$ years of age, $>30 \times 10^{6}$ leukocytes $/ \mu \mathrm{L}$, early $\mathrm{T}$ cell leukemia, Philadelphia chromosome, complex karyotype, and failure to induction treatment) [4]. Patients received either HyperCVAD [21] or an institutional protocol (1095 protocol) [22] with or without rituximab. Complete remission (CR) was defined as leukemia cells not detectable by light microscopy in peripheral blood or cerebrospinal fluid and $<5 \%$ blasts in a BM with trilineage hematopoiesis. To consider $\mathrm{CR}$, absolute neutrophil count (ANC) greater than $1.0 \times 10^{9} / \mathrm{L}$, platelet counts greater than $100 \times 10^{9} / \mathrm{L}$, and no recurrence should be observed for at least 4 weeks [23, 24]. Induction failure was defined as failure to achieve $\mathrm{CR}$ after first induction therapy; relapsed disease as the reappearance of blasts in blood or BM $(>5 \%)$. RFS was defined as the time elapsed from diagnosis to relapse and OS as the time elapsed from diagnosis to death by any cause or last follow up.

\section{Bone marrow fibrosis}

BM biopsies were evaluated in a blinded manner by one hematopathologist. Biopsy samples were fixed in formaldehyde, decalcified, and embedded in paraffin. Pathology material was discarded when samples were crushed, had extensive necrosis, where subcortical marrows and/or the evaluation area was less than three marrow spaces. Hematoxylin-eosin, reticulin, and Masson trichrome stains were performed. BM cellularity and blast count were estimated in all samples. Fibrosis was described using both modified Bauermeister scale as described by Bain et al. [25], and volume of reticulin/collagen fibers adapted from Nóren-Nyström et.al. [17]

Modified Bauermeister scale included four grades: grade 0 with no reticulin fibers demonstrable, grade 1 with occasional fine individual fibers and foci of a fine network, grade 2 with diffuse fiber network of fine fibers without coarse fibers, grade 3 with diffuse fiber network with scattered thick coarse fibers but no mature collagen, and grade 4 with diffuse coarse fiber network with areas of collagenization. Significant fibrosis was considered a modified Bauermeister scale $\geq$ grade 2 .

The volume of reticulin/collagen fibers for each sample was estimated using a 63-point graticule representing $25 \mathrm{mi}$ crons [2] at $\times 40$ magnification in 10 randomly selected fields within the marrow area and finally expressed as the average of this 10 selected fields per sample. The volume was determined by the percentage of the area occupied by graticule-crossing points or hits overlaying fibers versus hits over reference space (Fig. 1). A Lumera microscope camera and Infinity Analyze software version 6.4.1 was used. Significant or deep fibrosis reduction was considered $\geq 50 \%$ reduction of the volume of reticulin/collagen fibers after induction treatment.

Survival analyses were performed regarding (1) the presence or not of significant BM fibrosis at diagnosis, (2) the

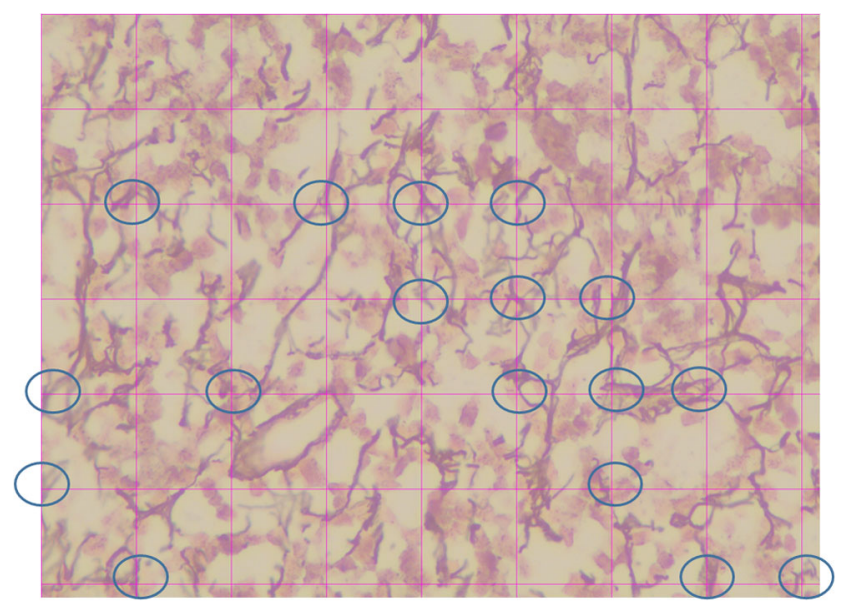

Fig. 1 Bone marrow biopsy stained with Masson trichrome stain. Twenty-five microns [2], $\times 40$. Sixty-three-point graticule. In this figure, there are 17 (of 63 total) graticule points crossed by collagen fibers representing $27 \%$ volume of collagen fibers of the field 
significant reduction of $\mathrm{BM}$ fibrosis post-induction treatment, and (3) the amount of BM fibrosis at diagnosis combined with the depth of BM fibrosis reduction post-induction treatment. For this third analysis, patients were classified into three groups: without significant fibrosis at diagnosis regardless of fibrosis reduction post-induction treatment (group 1), with significant fibrosis at diagnosis and a reduction of volume of reticulin/collagen fibers post-induction treatment of $<50 \%$ (group 2), and with significant fibrosis at diagnosis and reduction of volume of reticulin/collagen fibers post-induction treatment of $\geq 50 \%$ (group 3 ).

\section{Statistical analysis}

Continuous variables were described as medians and were compared with the Mann-Whitney $U$ test. Categorical variables were described as frequencies and proportions and were compared with $X^{2}$ and Fisher's exact test. Correlation analysis was performed using the Spearman correlation test. Survival analysis was performed with Kaplan-Meier curves and differences determined by log-rank test. Statistical significance was established at $p<0.05$ (two-tailed). All statistical analyses were conducted using SPSS 20.0 software program (Statistical Package for Social Sciences, SPSS Inc. Chicago, IL., USA).

\section{Results}

\section{Baseline characteristics}

A total of 44 patients were included in the study. Median age at diagnosis was 27 years (range, 17-67 years), 23 (52\%) were male, and $38(86 \%)$ patients had ECOG performance score of $0-1$. Regarding cytogenetic profile, $20(46 \%)$ patients had a normal karyotype, 1 (2\%) patient had complex karyotype ( $\geq 5$ chromosomal abnormalities), and Philadelphia chromosome was identified by FISH or karyotype in 10 (23\%) patients. There were no T or mature B cell leukemias, $41(93.2 \%)$ patients had pre-B cell leukemia and 28 (64\%) were considered high-risk. Additional baseline characteristics are listed in Table 1.

\section{Therapy and response}

A total of $27(61 \%)$ patients received HyperCVAD regimen with or without rituximab. Of the 10 patients with Philadelphia chromosome, $8(80 \%)$ received imatinib. During induction, $57 \%$ used granulocyte colony stimulating factor and $77 \%$ developed an infection. In terms of response assessment, $31(71 \%)$ patients achieved CR with induction therapy and 13 (29\%) failed induction. After 24 months follow up, $29(59 \%)$ patients relapsed; for the whole group, RFS was 10.6 months (IC 95\%, 7.08-14.07) and OS was 17.1 months (IC 95\%, 10.33-23.96).

\section{Bone marrow fibrosis assessment}

Bone marrow core biopsies had a median size of $1.3 \mathrm{~cm}$ (range, 0.7-3.4 cm) at diagnosis and $1.5 \mathrm{~cm}$ (range, 0.6$2.5 \mathrm{~cm}$ ) post-induction treatment. Median evaluable BM spaces at diagnosis were 11.5 (range, 5-40) and post induction, 10 (range, 4-21).

We correlated BM fibrosis description using the volume of reticulin/collagen fibers with the modified Bauermeister scale, both methods had a good correlation at diagnosis $(p \leq .001)$ and post-induction treatment $(p \leq .001)$ (Fig. 2). Since the modified Bauermeister scale is widely used for fibrosis description, we compared patients based on the presence ( $\geq$ grade 2 ) or absence (grade 0-1) of significant fibrosis at diagnosis (Table 1). Twenty-nine $(66 \%)$ patients had significant $\mathrm{BM}$ fibrosis at diagnosis, and there were no significant differences in baseline characteristics at diagnosis between patients with or without fibrosis. There were more high-risk patients $(55 \%$ vs $80 \%, p=.185)$ in the group without significant fibrosis. Median white blood cell count $\left(7.4 \times 10^{3} / \mu \mathrm{L}\right.$ vs $\left.20.7 \times 10^{3} / \mu \mathrm{L}, p=.154\right)$ and peripheral blast count $(25 \%$ vs $66 \%, p=.063)$ were lower in patients with significant fibrosis at diagnosis. These differences were not statistically significant.

The presence of significant BM fibrosis at diagnosis was not correlated with induction failure ( 6 patients vs 7 patients, $p=.313$ ) or OS (20.0 months vs 12.3 months, $p=.061)$. RFS was longer in patients with significant fibrosis at diagnosis (12.3 months vs 8.8 months, $p=.044)$, but the significance was lost when patients were classified according to risk (highrisk patients 9.4 months vs 8.2 months, $p=.291$; standard-risk patients 12.4 months vs 10.6 months, $p=.094$ ).

When Philadelphia positive patients were analyzed, the presence of significant BM fibrosis at diagnosis was not correlated with longer RFS (3.4 months vs 8.2 months, $p=.438$ ) or OS (5.6 months vs 10.8 months, $p=.600$ ). In Philadelphia negative patients, RFS (12.4 months vs 10.5 months, $p=.015)$ and OS (28.3 months vs 17.2 months, $p=.052)$ were longer in those with significant BM fibrosis at diagnosis.

Twenty $(45 \%)$ patients achieved a significant reduction in BM fibrosis post-induction treatment measured by volume of reticulin/collagen fibers, this was correlated to less induction failure ( 2 patients vs 11 patients, $p=.018$ ), longer RFS (12.1 months vs 10.5 months, $p=.037)$, and longer OS (28.3 months vs 13.1 months, $p=.028)$. When standard and high-risk patients were individually analyzed, the impact of $\mathrm{BM}$ fibrosis reduction post-induction treatment was lost for high-risk patients (RFS: 8.7 months vs 8.2 months, $p=.336$. OS: 14.1 months vs 12.1 months, $p=.600$ ) (Fig. 3 ). The significance was maintained in standard-risk patients (RFS: 19.1 months vs 10.6 months, $p=.022$. OS: 54.4 months vs 16.4 months, $p=.033$ ) (Fig. 4). 
Table 1 Demographics and characteristic of evaluable patients at diagnosis

\begin{tabular}{|c|c|c|c|c|}
\hline Characteristic & $\begin{array}{l}\text { All patients } \\
N=44\end{array}$ & $\begin{array}{l}\text { Significant fibrosis (Bauermeister } \geq 2 \text { ) } \\
N=29\end{array}$ & $\begin{array}{l}\text { Without significant fibrosis (Bauermeister }<2 \text { ) } \\
N=15\end{array}$ & $p$ \\
\hline Sex, $n(\%)$ & & & & $0.213 *$ \\
\hline Male & $23(52)$ & $13(45)$ & $10(67)$ & \\
\hline Female & $21(48)$ & $16(55)$ & $5(33)$ & \\
\hline Median age in years (range) & $27(17-67)$ & $26(17-67)$ & $28(18-56)$ & $0.907^{\dagger}$ \\
\hline ECOG, $n(\%)$ & & & & $0.277^{*}$ \\
\hline $0-1$ & $38(86)$ & $23(79)$ & $15(100)$ & \\
\hline $2-3$ & $6(14)$ & $6(21)$ & 0 & \\
\hline Karyotype, $n(\%)$ & & & & $0.636^{*}$ \\
\hline Philadelphia chromosome & $10(23)$ & $6(21)$ & $4(27)$ & \\
\hline Normal & $20(46)$ & $12(41)$ & $8(53)$ & \\
\hline Others & $3(7)$ & $2(7)$ & $1(7)$ & \\
\hline Not evaluable & $11(25)$ & $9(31)$ & $2(13)$ & \\
\hline Immunophenotype, $n(\%)$ & & & & $0.540^{*}$ \\
\hline Early pre-B & $3(6.8)$ & $3(10.3)$ & 0 & \\
\hline Pre-B & $41(93.2)$ & $26(89.7)$ & $15(100)$ & \\
\hline Risk, $n(\%)$ & & & & $0.185^{*}$ \\
\hline Standard & $16(36)$ & $13(45)$ & $3(20)$ & \\
\hline High & $28(64)$ & $16(55)$ & $12(80)$ & \\
\hline Induction treatment, $n(\%)$ & & & & $0.333 *$ \\
\hline HyperCVAD \pm Rituximab & $27(61)$ & $16(55)$ & $11(73)$ & \\
\hline Others & $17(39)$ & $13(45)$ & $4(27)$ & \\
\hline Infection at induction, $n(\%)$ & $34(77)$ & $23(79)$ & $11(73)$ & $0.714^{*}$ \\
\hline Hemoglobin (g/dL), & & & & $0.225^{\dagger}$ \\
\hline Median (range) & $8.1(4.1-15.3)$ & $7.7(4.1-12.4)$ & $8.8(6.5-15.3)$ & \\
\hline Leucocytes $\times 10^{3} / \mu \mathrm{L}$, & & & & $0.154^{\dagger}$ \\
\hline Median (range) & $9.7(1-384)$ & $7.4(1-384)$ & $20.7(1.9-272)$ & \\
\hline Platelets $\times 10^{9} / \mathrm{L}$, & & & & $0.682^{\dagger}$ \\
\hline Median (range) & $37.5(8-205)$ & $38(11-205)$ & $37(8-158)$ & \\
\hline Blasts in peripheral blood (\%), & & & & $0.063^{\dagger}$ \\
\hline Median (range) & $40(0-90)$ & $35(0-87)$ & $66(1-90)$ & \\
\hline Blasts in BM $(\%)$, & & & & $0.554^{\dagger}$ \\
\hline Median (range) & $64(20-95)$ & $64(20-95)$ & $68(36-91)$ & \\
\hline BM cellularity $(\%)$ & & & & $0.907^{\dagger}$ \\
\hline Median (range) & $88(20-100)$ & $90(20-100)$ & $90(60-100)$ & \\
\hline
\end{tabular}

$*$ Chi2, $†$ Mann-Whitney $U$

When Philadelphia positive patients were analyzed, a significant reduction in BM fibrosis after induction treatment was not correlated with longer RFS (8.4 months vs 8.2 months, $p=.702$ ) or OS (10.8 months vs 5.6 months, $p=.935)$. In Philadelphia negative patients, the reduction in BM fibrosis was positively correlated with RFS (19.1 months vs 10.5 months, $p=.003$ ) and OS (39.3 months vs 16.4 months, $p=.023$ ).

We did survival analysis regarding the amount of BM fibrosis at diagnosis combined with the depth BM fibrosis reduction post-induction treatment. Patients were classified into three groups: without significant fibrosis at diagnosis regardless of fibrosis reduction post-induction treatment (group 1), with significant fibrosis at diagnosis and a reduction of volume of reticulin/collagen fibers post-induction treatment of $<$ $50 \%$ (group 2), and with significant fibrosis at diagnosis and reduction of volume of reticulin/collagen fibers post-induction treatment of $\geq 50 \%$ (group 3). Patients in group 3 had a significantly better RFS (18 months) vs patients in group 1 (9 months) and group 2 (7 months); $p=.024$. OS was also better in patients in group 3 (39 months) vs groups 1 and 2 (12 months); $p=.01$ (Fig. 5). The 2-year RFS for patients in group 3 was $37 \%$ vs $12 \%$ for group 2 and $22 \%$ for group 1, and 2 -year OS for patients in group 3 was $60 \%$ vs $17 \%$ for group 2 and $9 \%$ for group 1. 
Fig. 2 Correlation of fibrosis description by volume of reticulin/collagen fibers and fibrosis by modified Bauermeister scale at diagnosis $(p \leq .001)$. We found the same correlation with $p \leq .001$ after induction treatment

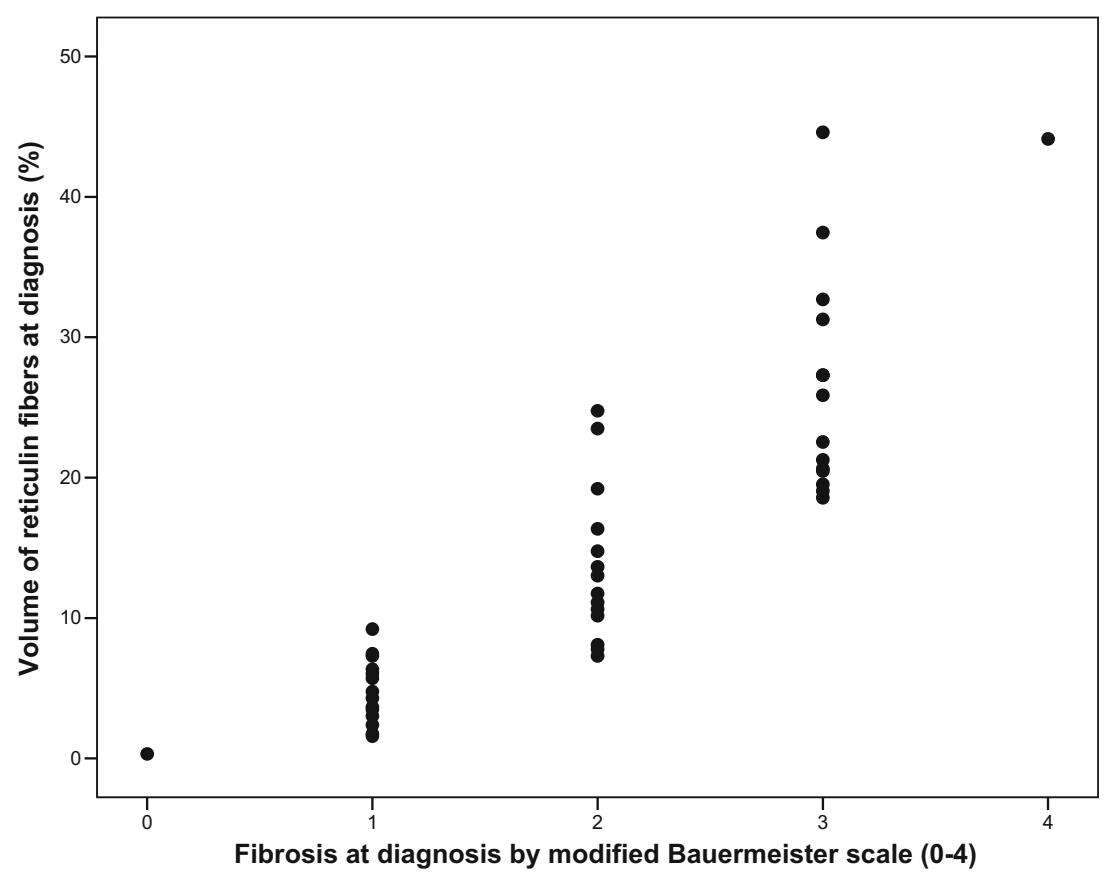

Treatment choice between HyperCVAD or an institutional protocol or the use of imatinib did not impact on BM fibrosis reduction post-induction.

The differences between diagnosis and post-induction BM fibrosis are shown in Table 2. Median volume of reticulin/ collagen fibers was $15.3 \%$ (range, $0.32 \%-44.6 \%$ ) at diagnosis and $9.5 \%$ (range, $0.16 \%-38.6 \%$ ) post-induction. After induction, none of the patients had grade 3 or 4 fibrosis, 19 (43\%) biopsies had grade 1 fibrosis and 22 (50\%) biopsies had grade 2 fibrosis.

When analyzed by a median change in volume of reticulin/ collagen fibers, we found that patients with normal karyotype had an incremental increase of $19.4 \%$ in BM fibrosis postinduction instead of the reduction seen in patients with other karyotypes.

\section{Discussion}

Bone marrow vascular and endosteal niches provide support for normal hematopoietic stem cells and control proliferation, differentiation, migration, and death [26, 27]. Leukemia cells (LCs) share the same interaction mechanisms as normal hematopoietic stem cells with marrow microenvironment, but somehow overcome apoptosis and replace normal cells [25, 28]. Previous reports on ALL confirm that at diagnosis, there is increased $\mathrm{BM}$ fibrosis and abnormal angiogenesis.

LCs interact through VLA-4 with VCAM-1 and fibronectin on stromal cells which then secrete TGF $\beta 1$ and BMP-6, cytokines associated with fibrosis. They also interact through CXCL12 with its receptor CXCR4 producing an elevation of IL-8 that promotes angiogenesis. This interaction with stromal cells inhibits LC apoptosis through the activation of Bcl-2 and Bcl-xL. [12, 13, 29, 30] LCs compete with normal hematopoietic stem cells for space in the marrow niche by secreting stem cell factor which inhibits normal cell interaction [30]. At diagnosis, serum concentration cytokines associated with fibrosis such as TNF $\alpha$, FGF $\beta$, HGF, and MMP-9 are high in patients with ALL. [31, 32] VEGF concentration is low at diagnosis and returns to normal after treatment suggesting that it may be consumed because of increased angiogenesis [33]. All these pathways ensure LC survival and proliferation.

Since Hann et al. in 1978 described the presence of BM fibrosis in $57 \%$ of children with ALL, every report in acute leukemia confirms that the stromal microenvironment is part of the pathophysiology of the disease [7, 16-18].

Wallis et al. described BM fibrosis in 63 children with ALL. [14] They did not find any association between the presence of BM fibrosis at diagnosis and karyotype, hemoglobin or platelet count. Patients with fibrosis had lower leukocyte counts at diagnosis and, after induction treatment, all but two BMs returned to normal.

Nath et al. also found an inverse correlation between BM fibrosis and leukocyte and blast counts, patients with significant fibrosis had lower leukocyte and blast counts in peripheral blood at diagnosis [30]. They described higher cellularity in patients with significant fibrosis and suggest this was because of trapped blasts in the fibrotic BM. They proposed that LCs create an environment that protects them from apoptosis, but this might be overcome by intensive chemotherapy.

In a letter to the editor of Leukemia Journal in 2005, NórenNyström et al. reported for the first time the negative prognostic impact of vascular density and BM fibrosis evaluated by 

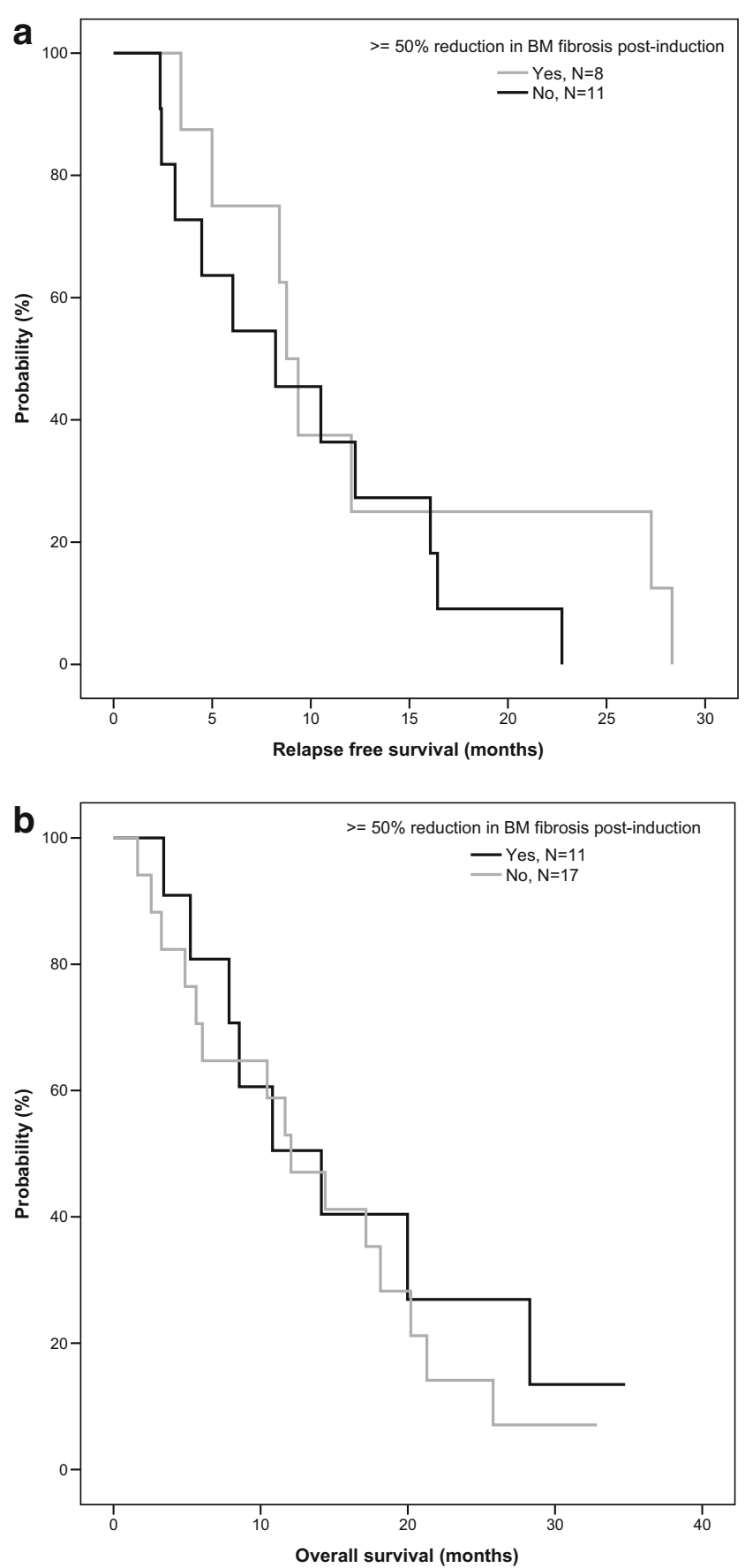

Fig. 3 Survival in high-risk patients a RFS: 8.8 months for patients with $\geq 50 \%$ reduction vs 8.2 months for those without, $p=.336$. b OS: 14.1 months for patients with $\geq 50 \%$ reduction vs 12.1 months for those without, $p=.600$

reticulin fiber density (RFD) in 81 children with ALL. [16] They found that patients with high-risk ALL had higher BM fibrosis at diagnosis and those patients relapsed more. In 2008, they published a complete report on BM fibrosis in 166 children [17]. Patients with B cell ALL had higher RFD compared with T cell ALL patients. They also found a negative correlation between RFD and leukocyte count. They did not find any
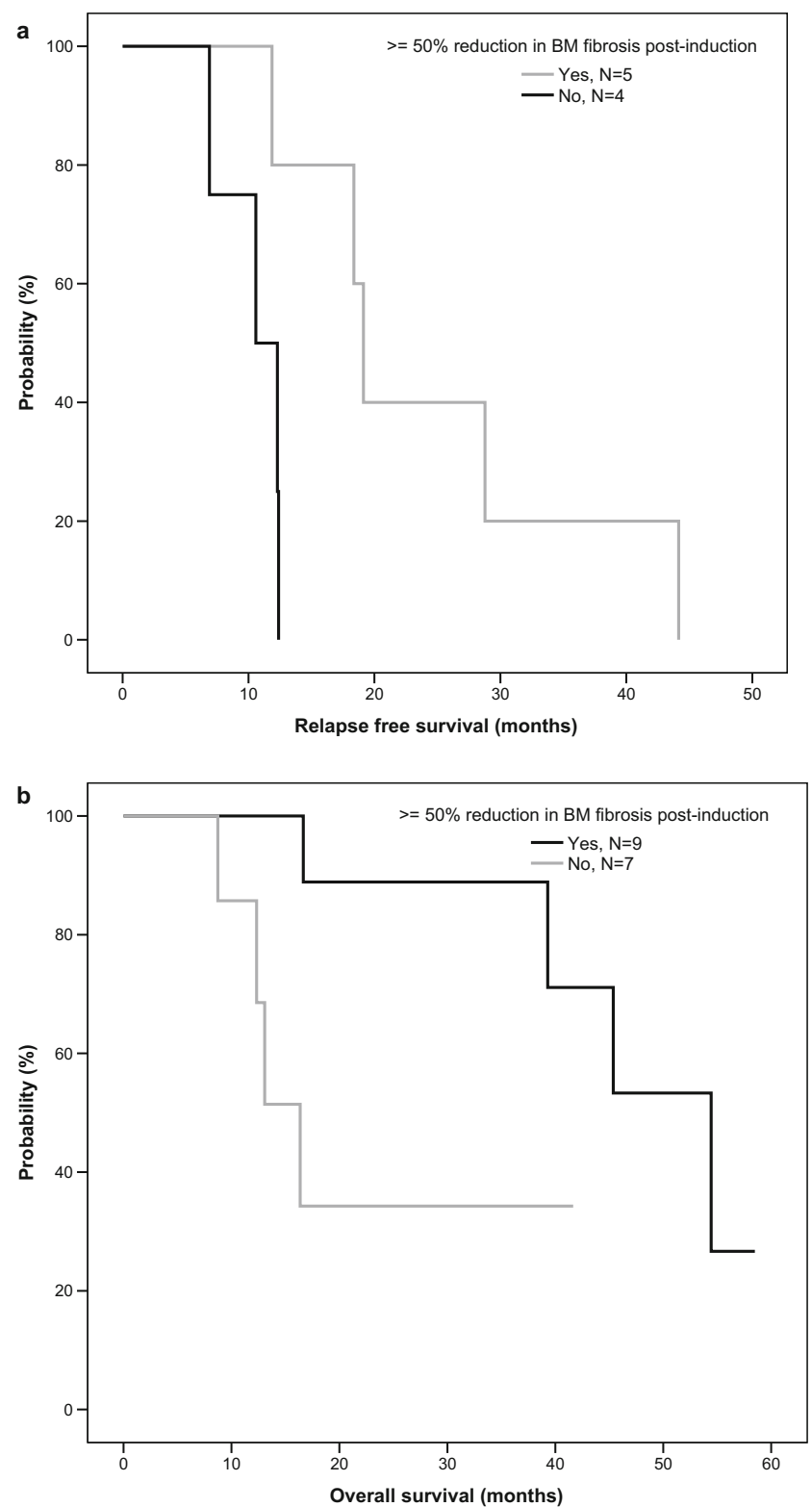

Fig. 4 Survival in standard-risk patients a RFS: 19.1 months for patients with $\geq 50 \%$ reduction vs 10.6 months for those without, $p=.022$. b OS: 54.4 months for patients with $\geq 50 \%$ reduction vs 16.4 months for those without, $p=.033$

other baseline characteristic (sex, risk, central nervous system disease, or karyotype) to be associated with higher RFD. In this second report, they only found a prognostic impact of RFD at diagnosis in low-risk patients, those with higher RFD had shorter RFS. Patients that achieved more than the median RFD reduction (>8.4\%) had better outcomes but it was only significant in those with low RFD at diagnosis.

In 2010, Bharos A et al. published contradicting results. They did not find any association between the presence of BM fibrosis at diagnosis and RFS or OS [15]. They described BM fibrosis with the modified Bauermeister scale and using RFD with good correlation between both methods $(p<.001)$. 

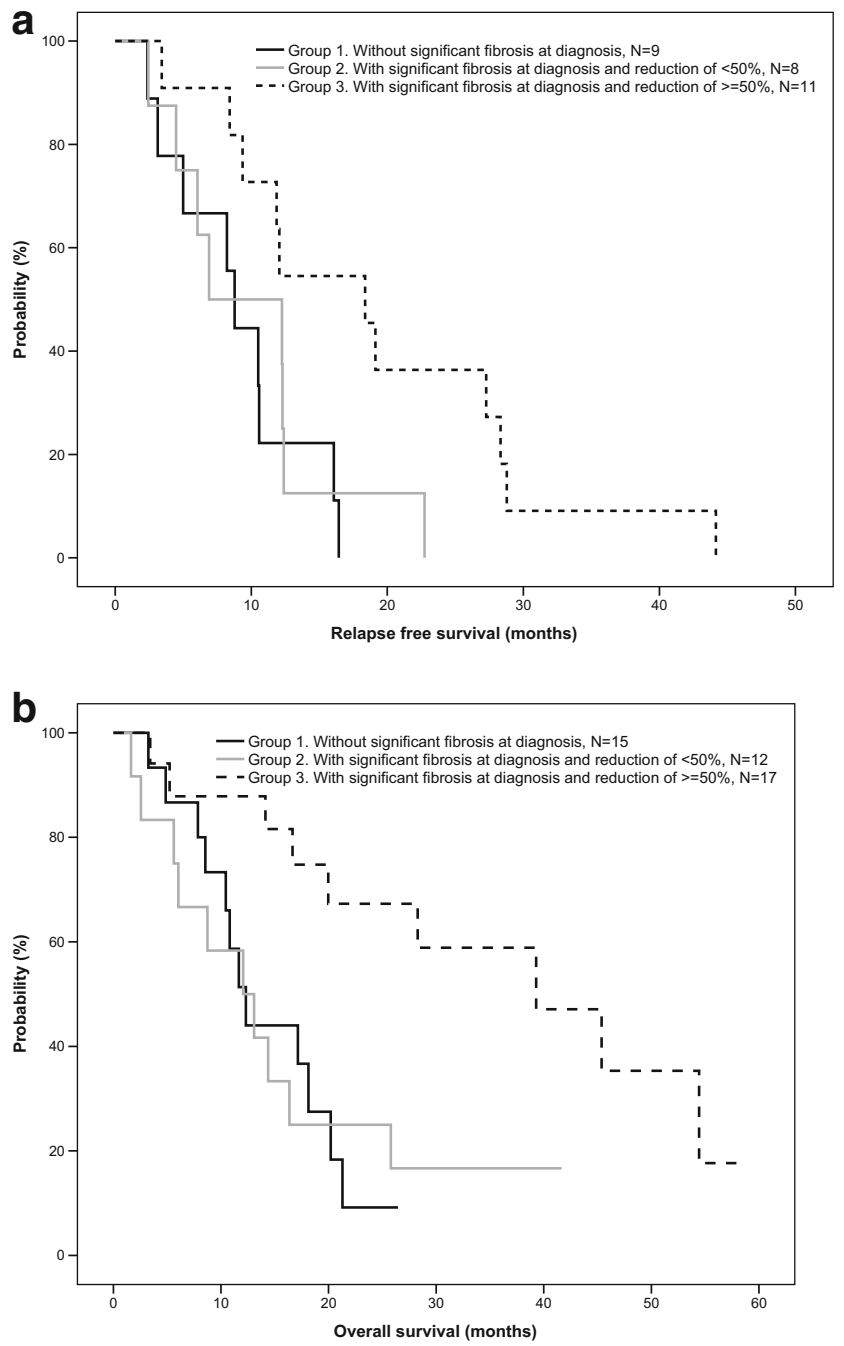

Fig. 5 Survival in patients classified in three group categories for fibrosis reduction. a RFS 18.4 months - group 3 vs 8.8 months - group 1 and 6.9 months - group 2, $p=.024$. b OS 39 months - group 3 vs 12 months for groups 1 and $2, p=.01$

They found that $2.5 \%$ of the patients had grade 0 fibrosis, $33.3 \%$ had grade 1, 37\% had grade 2, 19.7\% had grade 3, and $7.5 \%$ had grade 4 fibrosis. They evidenced a negative correlation between RFD and leukocyte and blast counts in peripheral blood at diagnosis as documented by previous authors.

In our study, $66 \%$ of the patients had significant fibrosis at diagnosis. We did not find any association between the presence or absence of significant fibrosis at diagnosis and any of the baseline characteristics such as sex, age, ECOG performance status, karyotype, immunophenotype, leukemia risk, induction treatment given, hemoglobin, or platelet count. Like Wallis et al. and Nath et al., we found that patients with significant fibrosis had lower leukocyte counts at diagnosis $(7.4 \times 10$ [3]/ $\mu \mathrm{L}$ vs $20.7 \times 10[3] / \mu \mathrm{L}, p=.125)$ and lower blast counts in peripheral blood (35\% vs $66 \%, p=.063$ ), but these did not reach statistical significance. We did not find any association between the presence of fibrosis at diagnosis and BM cellularity.
The presence of significant BM fibrosis at diagnosis was associated with longer RFS, but the significance was lost when patients were classified according to risk. Philadelphia negative patients with significant fibrosis at diagnosis had longer RFS. Most of the previous studies excluded these patients.

Patients who achieved a significant reduction in fibrosis post-induction treatment measured by the volume of reticulin/collagen fibers had less induction failure, longer RFS and OS in our study. The significance was only maintained in standard-risk and Philadelphia negative patients as described by the second report by Nóren-Nyström.

When classified by groups regarding the amount of BM fibrosis at diagnosis combined with the depth of BM fibrosis reduction post-induction treatment, we found that patients with significant fibrosis at diagnosis and reduction of volume of reticulin/collagen fibers post-induction treatment of $\geq 50 \%$ (group 3) had longer RFS and OS compared with those who did not have fibrosis at diagnosis regardless of its reduction (group 1) or patients who had fibrosis at diagnosis and did not achieve such a reduction (group 2).

In our study, $2.3 \%$ of patients had grade 0 fibrosis, $31.8 \%$ had grade $1,31.8 \%$ had grade $2,31.8 \%$ had grade 3 , and $1 \%$ had grade 4 fibrosis. After induction, fibrosis by volume of reticulin/collagen fibers reduced from $15.3 \%$ at diagnosis to $9.48 \%$ post-induction treatment. Thomas $\mathrm{X}$ et al. [26] reported more patients with grade 0 fibrosis $(36 \%)$ at diagnosis.

Patients with normal karyotype had an incremental increase in BM fibrosis post-induction. These might point to the fact that patients with normal karyotype might have other high-risk molecular abnormalities that we are not able to detect such as Philadelphia-like mutations and that these abnormalities could cause an increment in fibrosis [34, 35].

Our hypothesis is that the leukemia-stimulated stromal environment keeps the malignant cells in the BM initially and protects them from apoptosis through the interactions previously described. In vitro, these interactions make the LCs survive longer after exposition to chemotherapy [7, 13, 14]. This hypothesis would explain the lower WBC and peripheral blood blast counts in patients with significant BM fibrosis at diagnosis. Our findings suggest that in vivo, successful and intensive chemotherapy reduces fibrosis and overcomes the protection that the microenvironment brought to LCs in standard-risk and Philadelphia negative patients, but not in high-risk patients, including Philadelphia positive patients. Our results should be taken with care when evaluating the latter.

Although the Thiele et al. grading scale [36] is recommended by the WHO for the evaluation of BM fibrosis, this was reviewed mainly in the context of myeloproliferative neoplasms. Bauermeister scale modified by Bain et al. in 2001 has been previously used for fibrosis description in patients with ALL and was found to have good correlation with fibrosis description by volume of reticulin/collagen fibers [10, 15]. Both the modified Bauermeister and the Thiele et al. scale take into account 
Table 2 Bone marrow fibrosis described by volume of reticulin/collagen fibers and modified Bauermeister scale at diagnosis and post-induction

\begin{tabular}{lll}
\hline $\begin{array}{l}\text { Fibrosis } \\
\text { Volume of reticulin fibers }\end{array}$ & $\begin{array}{l}\text { Diagnosis } \\
\text { Median (range) }\end{array}$ & $\begin{array}{l}\text { Post-induction } \\
\text { Median (range) }\end{array}$ \\
\hline Whole group & $15.3(0.32-44.6)$ & $9.48(0.16-38.6)$ \\
Karyotype & $9.7(3.0-27.3)$ & $6.3(0.2-18.4)$ \\
$\quad$ Philadelphia chromosome & $11.4(1.6-44.1)$ & $10.6(1.9-38.6)$ \\
$\quad$ Normal & $19.5(0.3-24.8)$ & $3.0(0.6-8.7)$ \\
Others & $20.6(2.4-44.6)$ & $7.5(1.6-14.9)$ \\
$\quad$ Not evaluable & & $8.7(2.7-21.6)$ \\
Risk category & $19.5(4.3-32.7)$ & $6.7(0.16-38.6)$ \\
$\quad$ Standard-risk & $8.7(0.32-44.7)$ & $N(\%)$ \\
$\quad$ High-risk & $N(\%)$ & $3(6.8)$ \\
Fibrosis by modified Bauermeister scale & $1(2.3)$ & $19(43.2)$ \\
0 No reticulin fibers demonstrable & $14(31.8)$ & $22(50)$ \\
1 Occasional fine individual fibers & $14(31.8)$ & 0 \\
2 Fine fiber network throughout most of the section without coarse fibers & $14(31.8)$ & $1(2.3)$ \\
3 Diffuse fiber network with scattered thick coarse fibers but no mature collagen & 0 \\
4 Diffuse coarse network of fibers with areas of collagenization &
\end{tabular}

reticulin and collagen fibers [25], and we consider they could both be used for future description of fibrosis in ALL patients.

As a limitation of this study, we did not measure microvessel density (MVD) systematically using immunohistochemistry stain for von-Willebrand factor, CD34 or CD31. This has been described by some authors as a prognostic marker for survival in children with ALL and it is also an important way to differentiate BM fibrosis per se from perivascular fibrosis. Norén-Nyström et al. and PerezAtayde et al. reported that MVD was higher in T cell ALL, and when reticulin/collagen fiber description was combined with MVD, the survival curves dissected better, those with low reticulin/collagen fibrosis and low MVD had longer disease-free survival. [8, 18] Others have not found any correlation between higher MVD at diagnosis and survival. [9, 15] In all of these studies, MVD was increased at diagnosis at the expense of small microvessels, after induction treatment, MVD reduced and the vessels became larger.

Other limitations include the retrospective nature of this study, the lack of minimal residual disease measurement and the number of patients included. At our institution, minimal residual disease measurement was started in 2017.

The WHO recommends that BM biopsies be at least $1.5 \mathrm{~cm}$ in length so that $10 \mathrm{BM}$ spaces can be evaluated. In the interest of increasing patient sample, we used the inclusion criteria described by Norén-Nyström et al. were BM biopsies were accepted if they had more than three evaluable BM spaces.

We successfully reproduced the method of fibrosis estimation using the volume of reticulin/collagen fibers. Since this method is more time consuming and it has good correlation with the modified Bauermeister scale, we encourage the use of the latter or other widely accepted BM fibrosis description scales like the one described by Thiele et al. [36]

\section{Conclusions}

This study demonstrates that there is a role for BM fibrosis reduction as a prognostic factor in adult patients with ALL. That although having significant fibrosis at diagnosis does not clearly impact on outcomes, its reduction post-induction treatment has an impact on the survival of standard-risk patients. When we analyzed the amount of fibrosis at diagnosis combined with the depth of the reduction post-treatment, we found that the importance of fibrosis reduction lies on its presence at diagnosis and the capacity to reduce it with induction chemotherapy. Patients without fibrosis at diagnosis regardless of its reduction and patients who did not achieve a deep reduction of fibrosis post-treatment had worse survival. These results must be correlated with other prognostic factors like minimal residual disease and Philadelphia-like mutations.

Acknowledgments This work would not have been possible without the valuable contribution of all the laboratory personnel of the Department of Hematology and Pathology at the Instituto de Ciencias Medicas y Nutricion Salvador Zubiran in Mexico. We especially thank all our patients and their families.

\section{Compliance with ethical standards}

Conflict of interest The authors declare that they have no conflict of interest. 


\section{References}

1. Howlader N, Noone A, Krapcho M, et al. (2017) SEER cancer statistics review, 1975-2014, National Cancer Institute. Bethesda, MD, http://seer.cancer.gov/csr/1975 2014/, based on November 2016 SEER data submission, posted to the SEER web site, April Http://SeerCancerGov/Csr/1975 2014/. 2017:1-101. Accessed November 2017

2. Katz AJ, Chia VM, Schoonen WM, Kelsh MA (2015) Acute lymphoblastic leukemia: an assessment of international incidence, survival, and disease burden. Cancer Causes Control 26(11):16271642. https://doi.org/10.1007/s10552-015-0657-6

3. Curran E, Stock W (2015) How I treat acute lymphoblastic leukemia in older adolescents and young adults. Blood. 125(24):37023710. https://doi.org/10.1182/blood-2014-11-551481

4. National Comprehensive Cancer Network NCCN (2016) Guidelines Version 1. Acute lymphoblastic leukemia. NCCN Clinical Practice Guidelines in Oncology (NCCN Guidelines®). https://www.nccn.org/professionals/physician_gls/pdf/all.pdf. Accessed November 2017

5. Crespo-Solis E, Espinosa-Bautista K, Alvarado-Ibarra M, RozenFuller E, Pérez-Rocha F, Nava-Gómez C, Ortiz-Zepeda M, Álvarez-Vera JL, Ramos-Peñafiel CO, Meillón-García LA, Rodríguez-Rodríguez S, Pomerantz-Okon A, TurrubiatesHernández FJ, Demichelis-Gómez R (2018) Retrospective study of adults with acute lymphoid leukemia in Mexico City: first report of the working Group of Acute Leukemia (GTLA). Cancer Med 7(6):2423-2433. https://doi.org/10.1002/cam4.15132017.

6. Ru YX, Zhao SX, Liu EB, Yang QY, Liu JH, Pang TX, Chen HS (2007) Ultrastructural characteristics of bone marrow in patients with hematological disease: a study of 13 cases. Ultrastruct Pathol 31(5):327-332. https://doi.org/10.1080/01913120701643637

7. Hann IM, Evans DI, Marsden HB, Jones PM, Palmer MK (1978) Bone marrow fibrosis in acute lymphoblastic leukaemia of childhood. J Clin Pathol 31(4):313-315

8. Perez-Atayde AR, Sallan SE, Tedrow U, Connors S, Allred E, Folkman J (1997) Spectrum of tumor angiogenesis in the bone marrow of children with acute lymphoblastic leukemia. Am J Pathol 150(3):815-821

9. Pulè MA, Gullmann C, Dennis D, McMahon C, Jeffers M, Smith OP (2002) Increased angiogenesis in bone marrow of children with acute lymphoblastic leukaemia has no prognostic significance. Br J Haematol 118(4):991-998. https://doi.org/10.1046/j.1365-2141. 2002.03761.x

10. Nguyen T-V, Melville A, Nath S, Story C, Howell S, Sutton R, Zannettino A, Revesz T (2015) Bone marrow recovery by morphometry during induction chemotherapy for acute lymphoblastic leukemia in children. PLoS One 10(5):e0126233. https://doi.org/ 10.1371/journal.pone.0126233

11. Belurkar S, Nepali PB, Manandhar B, Manohar C (2015) Evaluation of post chemotherapy bone marrow changes in acute Leukaemia. Int J Sci Res Publ 5(1):2250-3153 www.ijsrp.org

12. Mudry RE, Fortney JE, York T, Hall BM, Gibson LF (2000) Stromal cells regulate survival of B-lineage leukemic cells during chemotherapy. Blood. 96(5):1926-1932 http://www.ncbi.nlm.nih. gov/pubmed/10961896

13. Døsen-Dahl G, Munthe E, Nygren MK, Stubberud H, Hystad ME, Rian E (2008) Bone marrow stroma cells regulate TIEG1 expression in acute lymphoblastic leukemia cells: role of TGF $\beta /$ BMP-6 and TIEG1 in chemotherapy escape. Int J Cancer 123(12):27592766. https://doi.org/10.1002/ijc.23833

14. Wallis JP, Reid MM (1989) Bone marrow fibrosis in childhood acute lymphoblastic leukaemia. J Clin Pathol 42(12):1253-1254. https://doi.org/10.1136/jcp.42.12.1253
15. Bharos A, Jong AJD, Manton $\mathrm{N}$ et al (2010) Bone marrow fibrosis and vascular density lack prognostic significance in childhood acute lymphoblastic leukaemia. Leukemia. 24(8):1537-1538. https://doi. org/10.1038/leu.2010.134

16. Norén-Nyström U, Roos G, Bergh A, Forestier E (2005) Prognostic impact of vascular density and fibrosis in the bone marrow of children with high-risk acute lymphoblastic leukemia [6]. Leukemia. 19(11):1998-2001. https://doi.org/10.1038/sj.leu.2403953

17. Norén-Nyström U, Roos G, Bergh A, Botling J, Lönnerholm G, Porwit A, Heyman M, Forestier E (2008) Bone marrow fibrosis in childhood acute lymphoblastic leukemia correlates to biological factors, treatment response and outcome. Leukemia. 22(3):504510. https://doi.org/10.1038/sj.leu.2405072

18. Norén-Nyström U, Heyman M, Frisk P, Golovleva I, Sundström C, Porwit A, Roos G, Bergh A, Forestier E (2009) Vascular density in childhood acute lymphoblastic leukaemia correlates to biological factors and outcome. Br J Haematol 146(5):521-530. https://doi. org/10.1111/j.1365-2141.2009.07796.x

19. Arber DA, Orazi A, Hasserjian R, Thiele J, Borowitz MJ, le Beau MM, Bloomfield CD, Cazzola M, Vardiman JW (2016) The 2016 revision to the World Health Organization classification of myeloid neoplasms and acute. Leukemia. 127:2391-2405. https://doi.org/ 10.1182/blood-2016-03-643544

20. Campo E, Swerdlow SH, Harris NL, Pileri S, Stein H, Jaffe ES (2008) WHO classification of tumors of hematopoietic and lymphoid tissues. Lyon 117:5019-5032. https://doi.org/10.1182/blood2011-01-293050

21. Kantarjian HM, O'Brien S, Smith TL et al (2000) Results of treatment with hyper-CVAD, a dose-intensive regimen, in adult acute lymphocytic leukemia. J Clin Oncol 18(3):547-561

22. Arteaga-Ortiz L, Buitrón-Santiago N, Rosas-López A, RosasArzate G, Armengolt-Jiménez A, Aguayo A, López-Karpovitch X, Crespo-Solís E (2008) Experiencia del INCMNSZ en pacientes adultos con leucemia linfoide aguda. Cohorte 2003-2007 con esquemas de tratamiento Hiper-CVAD y Protocolo 0195. Rev Investig Clin 60(6):459-469

23. Hoelzer D, Bassan R, Dombret H, Fielding A, Ribera JM, Buske C (2016) Acute lymphoblastic leukaemia in adult patients: ESMO clinical practice guidelines for diagnosis, treatment and follow-up. Ann Oncol 27(March):v69-v82. https://doi.org/10.1093/annonc/ mdw025

24. NCCN Guidelines Version 2.2016 Acute lymphoblastic leukemia.

25. Kuter DJ, Bain B, Mufti G, Bagg A, Hasserjian RP (2007) Bone marrow fibrosis: pathophysiology and clinical significance of increased bone marrow stromal fibres. Br J Haematol 139(3):351362. https://doi.org/10.1111/j.1365-2141.2007.06807.x

26. Arber DA, Orazi A, Hasserjian R et al (2015) Interaction of bone marrow stromal cells with lymphoblasts and effects of predinsolone on cytokine expression. Ann Hematol 1863(3):327-332. https:// doi.org/10.1007/s00277-005-0042-8

27. Leiva $\mathrm{O}, \mathrm{Ng} \mathrm{SK}$, Chitalia S, Balduini A, Matsuura S, Ravid K (2017) The role of the extracellular matrix in primary myelofibrosis. Blood Cancer J 7(2):1-9. https://doi.org/10.1038/bcj.2017.6

28. Tabe Y, Konopleva M (2014) Advances in understanding the leukemia microenvironment. Br J Haematol 164(6):767-778. https:// doi.org/10.1111/bjh.12725.ADVANCES

29. Ayala F, Dewar R, Kieran M, Kalluri R (2009) Contribution of bone microenvironment to leukemogenesis and leukemia progression. Leukemia. 23(12):2233-2241. https://doi.org/10.1038/leu.2009.175

30. Nath SV, Nicholson I, Tapp H, Zola H, Zannettino ACW, Revesz T (2011) Reticulin fibres anchor leukaemic blasts in the marrow of patients with acute lymphoblastic leukaemia. Med Hypotheses 77(3):333-335. https://doi.org/10.1016/j.mehy.2011.05.007

31. Aguayo A, Kantarjian H, Manshouri T, Gidel C, Estey E, Thomas D, Koller C, Estrov Z, O'Brien S, Keating M, Freireich E, Albitar M 
(2000) Angiogenesis in acute and chronic leukemias and myelodysplastic syndromes. Blood. 96(6):2240-2245

32. Aref S, Salama O, Shamaa S, El-Refaie M, Mourkos H (2007) Angiogenesis factor pattern differs in acute lymphoblastic leukemia and chronic lymphocytic leukemia. Hematology. 12(4):319-324. https://doi.org/10.1080/10245330701340759

33. Faderl S, Do K, Johnson MM et al (2005) Angiogenic factors may have a different prognostic role in adult acute lymphoblastic leukemia. Survival (Lond) 106(13):4303-4307. https://doi.org/10.1182/ blood-2005-03-1010.Reprints

34. Roberts KG, Li Y, Turner DP et al (2014) Targetable kinaseactivating lesions in Ph-like acute lymphoblastic leukemia. N Eng1 J Med 371(11):1005-1015. https://doi.org/10.1056/ NEJMoa1403088.

35. Herold T, Schneider S, Metzeler KH, Neumann M, Hartmann L, Roberts KG, Konstandin NP, Greif PA, Bräundl K, Ksienzyk B,
Huk N, Schneider I, Zellmeier E, Jurinovic V, Mansmann U, Hiddemann W, Mullighan CG, Bohlander SK, Spiekermann K, Hoelzer D, Brüggemann M, Baldus CD, Dreyling M, Gökbuget N (2017 Jan) Adults with Philadelphia chromosome - like acute lymphoblastic leukemia frequently have IGH-CRLF2 and JAK2 mutations, persistence of minimal residual disease and poor prognosis. Haematologica. 102(1):130-138. https://doi.org/10.3324/ haematol.2015.136366

36. Thiele J, Kvasnicka HM, Facchetti F, Franco V, Van Der WJ, Orazi A (2005) European consensus on grading bone marrow fibrosis and assessment of cellularity. Haematologica. 90:1128-1132

Publisher's note Springer Nature remains neutral with regard to jurisdictional claims in published maps and institutional affiliations. 